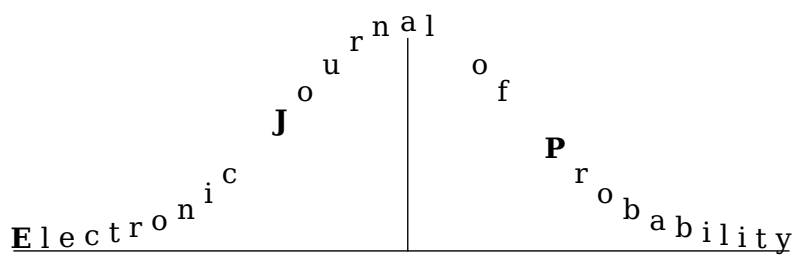

Electron. J. Probab. 26 (2021), article no. 38, 1-21.

ISSN: 1083-6489 https://doi.org/10.1214/21-EJP600

\title{
Asymptotic windings of the block determinants of a unitary Brownian motion and related diffusions*
}

\author{
Fabrice Baudoin $^{\dagger} \quad$ Jing Wang $^{\ddagger}$
}

\begin{abstract}
We study several matrix diffusion processes constructed from a unitary Brownian motion. In particular, we use the Stiefel fibration to lift the Brownian motion of the complex Grassmannian to the complex Stiefel manifold and deduce a skew-product decomposition of the Stiefel Brownian motion. As an application, we prove asymptotic laws for the determinants of the block entries of the unitary Brownian motion.
\end{abstract}

Keywords: asymptotic windings; asymptotic stochastic area; block determinants; Stiefel Brownian motion; Brownian motion of complex Grassmannian manifold.

MSC2020 subject classifications: 60J60; 60B20; 60J35.

Submitted to EJP on October 1, 2020, final version accepted on March 3, 2021.

\section{Contents}

1 Introduction

2 Some diffusions related to unitary Brownian motion 3

2.1 Stiefel fibration . . . . . . . . . . . . . . . . 3

2.2 Inhomogeneous coordinates on $G_{n, k} \ldots \ldots \ldots \ldots$

2.3 Brownian motions on $\widehat{G}_{n, k}$ and $V_{n, k} \ldots \ldots \ldots \ldots \ldots$

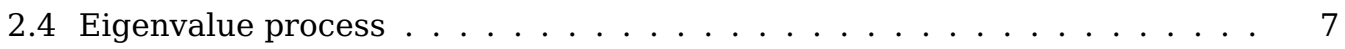

3 Skew-product decomposition of the Brownian motion of the Stiefel fibration 8

3.1 Connection form and horizontal Brownian motion on $V_{n, k} \ldots \ldots$

3.2 Skew-product decomposition of the Stiefel Brownian motion . . . . . . . 10

${ }^{*}$ F.B. was partly supported by the NSF grant DMS 1901315. J.W. was partly supported by the NSF grant DMS 1855523

${ }^{\dagger}$ University of Connecticut, Connecticut, U.S.A. E-mail: fabrice. baudoin@uconn .edu

${ }^{\ddagger}$ Purdue University, Indiana, U.S.A. E-mail: jingwang@purdue. edu 
Asymptotic windings of the block determinants of a unitary Brownian motion

4 Limit theorems $\quad 11$

4.1 Main limit theorem . . . . . . . . . . . . . . . . . . . . . . . . 11

4.2 Asymptotics of a generalized stochastic area . . . . . . . . . . . . . 16

4.3 Asymptotic windings . . . . . . . . . . . . . . . . . . . 17

4.4 The case $k \geq n-k \ldots \ldots \ldots \ldots \ldots \ldots$

4.5 On the moments of $\operatorname{det} Z_{t} \ldots \ldots \ldots \ldots \ldots \ldots$

$\begin{array}{lr}\text { References } & 20\end{array}$

\section{Introduction}

The Brownian motion on the Lie group of complex unitary matrices, in short unitary Brownian motion, is an extensively studied object in random matrix theory (see for instance [8, 23] and references therein). In this paper we study several diffusion processes naturally associated to this Brownian motion and show how they can be used to compute the exact or asymptotic distribution of some functionals. In particular, we emphasize the role of the Stiefel fibration over the complex Grassmannian space as an effective computational tool. As an application of our methods we obtain for instance the following result.

Theorem 1.1. Let

$$
U_{t}=\left(\begin{array}{cc}
X_{t} & Y_{t} \\
Z_{t} & W_{t}
\end{array}\right)
$$

be a Brownian motion on the unitary group $\mathbf{U}(n)$ with $Z_{t} \in \mathbb{C}^{k \times k}, 1 \leq k \leq n-1$. Assume that $\operatorname{det} Z_{0} \neq 0$. One has then the polar decomposition

$$
\operatorname{det}\left(Z_{t}\right)=\varrho_{t} e^{i \theta_{t}}
$$

where $0<\varrho_{t} \leq 1$ is a continuous semimartingale such that, in distribution, when $t \rightarrow+\infty$,

$$
\varrho_{t}^{2} \rightarrow \prod_{j=1}^{\min (k, n-k)} \mathfrak{B}_{j, \max (k, n-k)}
$$

where $\mathfrak{B}_{a, b}$ are independent beta random variables with parameters $(a, b)$ and $\theta_{t}$ is a realvalued continuous martingale such that the following convergence holds in distribution when $t \rightarrow+\infty$

$$
\frac{\theta_{t}}{t} \rightarrow \mathcal{C}_{k(n-k)}
$$

where $\mathcal{C}_{k(n-k)}$ is a Cauchy distribution of parameter $k(n-k)$.

The study of the limit law of $\varrho_{t}$ can be deduced from known results about the complex Jacobi ensemble, see [26]. Therefore, our main contribution lies in the study of the winding process $\theta_{t}$. We note that for $k=1$, the theorem therefore yields a Spitzer's type theorem (see [27]) for the windings of each of the entries of a unitary Brownian motion (the choice of bottom left corner block for $Z$ is arbitrary). Overall, the proof of Theorem 1.1 is rather long. An important step, interesting in itself, is to obtain the representation

$$
\theta_{t}=\theta_{0}+i \operatorname{tr}\left(D_{t}\right)+\int_{w[0, t]} \alpha
$$

where $D_{t}$ is a Brownian motion on the Lie algebra $\mathfrak{u}(k), w$ is a Brownian motion on the complex Grassmannian manifold $G_{n, k}$ and $\alpha$ is an almost everywhere defined one-form on $G_{n, k}$ whose exterior derivative yields the Kähler form of $G_{n, k}$. To study the functional $\int_{w[0, t]} \alpha$ which might be interpreted as a generalized stochastic area process in the sense 
Asymptotic windings of the block determinants of a unitary Brownian motion

of [6], we prove that $\int_{w[0, t]} \alpha$ is a time-changed Brownian motion with independent clock $\int_{0}^{t} \operatorname{tr}\left(w_{s}^{*} w_{s}\right) d s$ and finally study the distribution of this latter additive functional using a Girsanov transform in the spirit of $[6,13,30]$.

The paper is structured as follows. Section 2 mostly collects preliminary results. We present the geometry of the Stiefel fibration over the complex Grassmannian space, introduce a convenient set of coordinates and show how to obtain representations of the Brownian motions on the Stiefel and complex Grassmannian spaces from a unitary Brownian motion, see Theorem 2.1. We then study some related eigenvalue processes. In Section 3, we take advantage of the Stiefel fibration over the complex Grassmannian space to explicitly write a skew-product decomposition of the Stiefel Brownian motion. Section 4 is devoted to the study of limit laws of some functionals of the unitary Brownian motion and culminates with the proof of Theorem 1.1

\section{Notations:}

- If $M \in \mathbb{C}^{n \times n}$ is a $n \times n$ matrix with complex entries, we will denote $M^{*}=\bar{M}^{T}$ its adjoint.

- If $z_{i}=x_{i}+i y_{i}$ is a complex coordinate system, let

$$
\frac{\partial}{\partial z_{i}}=\frac{1}{2}\left(\frac{\partial}{\partial x_{i}}-i \frac{\partial}{\partial y_{i}}\right), \quad \frac{\partial}{\partial \bar{z}_{i}}=\frac{1}{2}\left(\frac{\partial}{\partial x_{i}}+i \frac{\partial}{\partial y_{i}}\right) .
$$

- Throughout the paper we work on a filtered probability space $\left(\Omega,\left(\mathcal{F}_{t}\right)_{t \geq 0}, P\right)$ that satisfies the usual conditions.

- If $X$ and $Y$ are semimartingales, we denote $\int X d Y$ the Itô integral, $\int X \circ d Y$ the Stratonovich integral and $\int d X d Y$ or $\langle X, Y\rangle$ the quadratic covariation.

- For matrix-valued semimartingales $M$ and $N$, the quadratic variation $\int d M d N$ is a matrix such that $\left(\int d M d N\right)_{i j}=\sum_{\ell} \int d M_{i \ell} d N_{\ell j}$.

- If $M$ is a semimartingale and $\eta$ a one-form, then $\int_{M[0, t]} \eta$ denotes the Stratonovich line integral of $\eta$ along the paths of $M$.

\section{Some diffusions related to unitary Brownian motion}

\subsection{Stiefel fibration}

Let $n \in \mathbb{N}, n \geq 2$, and $k \in\{1, \ldots, n\}$. The complex Stiefel manifold $V_{n, k}$ is the set of unitary $k$-frames in $\mathbb{C}^{n}$. In matrix notation we have

$$
V_{n, k}=\left\{M \in \mathbb{C}^{n \times k} \mid M^{*} M=I_{k}\right\} .
$$

As such $V_{n, k}$ is therefore an algebraic compact embedded submanifold of $\mathbb{C}^{n \times k}$ and inherits from $\mathbb{C}^{n \times k}$ a Riemannian structure. We note that $V_{n, 1}$ is isometric to the unit sphere $\mathbb{S}^{2 n-1}$. There is a right isometric action of the unitary group $\mathbf{U}(k)$ on $V_{n, k}$, which is simply given by the right matrix multiplication: $M g, M \in V_{n, k}, g \in \mathbf{U}(k)$. The quotient space by this action $G_{n, k}:=V_{n, k} / \mathbf{U}(k)$ is the complex Grassmannian manifold. It is a compact manifold of complex dimension $k(n-k)$. We note that $G_{n, k}$ can be identified with the set of $k$-dimensional subspaces of $\mathbb{C}^{n}$. In particular $G_{n, 1}$ is the complex projective space $\mathbb{C} P^{n-1}$. Since $G_{n, k}$ and $G_{n, n-k}$ can be identified with each other via orthogonal complement, without loss of generality we will therefore assume throughout the paper that $k \leq n-k$, except when explicitly mentioned otherwise (see section 4.4).

Let us quickly comment on the Riemannian structure of $G_{n, k}$ that we will be using and that is induced from the one of $V_{n, k}$. From Example 2.3 in [4], there exists a unique Riemannian metric on $G_{n, k}$ such that the projection map $\pi: V_{n, k} \rightarrow G_{n, k}$ is a 
Riemannian submersion. From Example 2.5 in [4] and Theorem 9.80 in [7] the fibers of this submersion are totally geodesic submanifolds of $V_{n, k}$ which are isometric to $\mathbf{U}(k)$. This therefore yields a fibration:

$$
\mathbf{U}(k) \rightarrow V_{n, k} \rightarrow G_{n, k}
$$

which is often referred to as the Stiefel fibration, see [3, 22]. We note that for $k=1$ it is nothing else but the classical Hopf fibration considered from the probabilistic viewpoint in [6]:

$$
\mathbf{U}(1) \rightarrow \mathbb{S}^{2 n-1} \rightarrow \mathbb{C} P^{n-1} .
$$

For further details on the Riemannian geometry of the complex Grassmannian manifolds we also refer to $[28,29]$, see in particular Theorem 4 in [28].

\subsection{Inhomogeneous coordinates on $G_{n, k}$}

We consider the open set $\widehat{V}_{n, k} \subset V_{n, k}$ given by

$$
\widehat{V}_{n, k}=\left\{\left(\begin{array}{l}
X \\
Z
\end{array}\right) \in V_{n, k}, \operatorname{det} Z \neq 0\right\}
$$

and the smooth map $p: \widehat{V}_{n, k} \rightarrow \mathbb{C}^{(n-k) \times k}$ given by $p\left(\begin{array}{l}X \\ Z\end{array}\right)=X Z^{-1}$. It is clear that for every $g \in \mathbf{U}(k)$ and $M \in V_{n, k}, p(M g)=p(M)$. Since $p$ is a submersion from $\widehat{V}_{n, k}$ onto its image $p\left(\widehat{V}_{n, k}\right)=\mathbb{C}^{(n-k) \times k}$ we deduce that there exists a unique diffeomorphism $\Psi$ from an open set of $G_{n, k}$ onto $\mathbb{C}^{(n-k) \times k}$ such that

$$
\Psi \circ \pi=p .
$$

The map $\Psi$ induces a (local) coordinate chart on $G_{n, k}$ that we call inhomogeneous by analogy with the case $k=1$ which corresponds to the complex projective space. The Riemannian metric of $G_{n, k}$ is then transported to $\mathbb{C}^{(n-k) \times k}$ using the map $\Psi$. In the sequel, we will denote $\mathbb{C}^{(n-k) \times k}$ endowed with this Riemannian metric by $\widehat{G}_{n, k}$ in order to emphasize the Riemannian structure that is used. Note that by construction $\widehat{G}_{n, k}$ is isometric to an open subset of $G_{n, k}$ and that it differs from $G_{n, k}$ by the sub-manifold at $\infty$, $\operatorname{det}(Z)=0$. In the case $n=2, k=1, G_{n, k}=\mathbb{C P}^{1}$ and the above description corresponds to the classical one-point compactification description $\mathbb{C P}^{1}=\mathbb{C} \cup\{\infty\}$. We note that the Stiefel fibration

$$
\mathbf{U}(k) \rightarrow V_{n, k} \rightarrow G_{n, k}
$$

yields a fibration

$$
\mathbf{U}(k) \rightarrow \widehat{V}_{n, k} \rightarrow \widehat{G}_{n, k}
$$

that we still refer to as the Stiefel fibration. The projection map $p: \widehat{V}_{n, k} \rightarrow \widehat{G}_{n, k}$, $\left(\begin{array}{l}X \\ Z\end{array}\right) \rightarrow X Z^{-1}$ is then a Riemannian submersion with totally geodesic fibers isometric to $\mathbf{U}(k)$.

\subsection{Brownian motions on $\widehat{G}_{n, k}$ and $V_{n, k}$}

In this section, we show how the Brownian motions on $\widehat{G}_{n, k}$ and $V_{n, k}$ can be constructed from a Brownian motion on the unitary group $\mathbf{U}(n)$. In the following, we will use the block notations as below: For any $U \in \mathbf{U}(n)$ and $A \in \mathfrak{u}(n)$ we will write

$$
U=\left(\begin{array}{cc}
X & Y \\
Z & V
\end{array}\right), \quad A=\left(\begin{array}{ll}
\alpha & \beta \\
\gamma & \epsilon
\end{array}\right)
$$


Asymptotic windings of the block determinants of a unitary Brownian motion

where $X, \gamma \in \mathbb{C}^{(n-k) \times k}, Y, \epsilon \in \mathbb{C}^{(n-k) \times(n-k)}, Z, \alpha \in \mathbb{C}^{k \times k}, V, \beta \in \mathbb{C}^{k \times(n-k)}$. We recall that the Lie algebra $\mathfrak{u}(n)$ consists of all skew-Hermitian matrices

$$
\mathfrak{u}(n)=\left\{X \in \mathbb{C}^{n \times n} \mid X=-X^{*}\right\},
$$

which we equip with the inner product $\langle X, Y\rangle_{\mathfrak{u}(n)}=-\frac{1}{2} \operatorname{tr}(X Y)$. This induces a Riemannian metric on $\mathbf{U}(n)$. Consider now on $\mathfrak{u}(n)$ a Brownian motion $\left(A_{t}\right)_{t \geq 0}$ and the matrix-valued process $\left(U_{t}\right)_{t \geq 0}$ that satisfy the Stratonovich stochastic differential equation:

$$
\left\{\begin{array}{l}
d U_{t}=U_{t} \circ d A_{t}, \\
U_{0}=\left(\begin{array}{ll}
X_{0} & Y_{0} \\
Z_{0} & V_{0}
\end{array}\right), \operatorname{det} Z_{0} \neq 0 .
\end{array}\right.
$$

The process $\left(U_{t}\right)_{t \geq 0}$ is a Brownian motion on $\mathbf{U}(n)$ (which is not started from the identity). If we write the block decomposition

$$
U_{t}=\left(\begin{array}{ll}
X_{t} & Y_{t} \\
Z_{t} & V_{t}
\end{array}\right)
$$

then, it is known that both of the processes $X^{*} X$ and $Z^{*} Z=I_{k}-X^{*} X$ belong to the wellknown family of (complex) matrix Jacobi processes that have already been extensively studied in the literature, see for instance [9], [11], [13], [19] and [20]. In particular, one can see that $Z^{*} Z$ satisfies

$$
d\left(Z^{*} Z\right)=\sqrt{Z^{*} Z} d \mathbf{B} \sqrt{I_{k}-Z^{*} Z}+\sqrt{I_{k}-Z^{*} Z} d \mathbf{B}^{*} \sqrt{Z^{*} Z}+\left(2 k I_{k}-2 n Z^{*} Z\right) d t
$$

where $\mathbf{B}$ is a $\mathbb{C}^{k \times k}$ Brownian motion.

Theorem 2.1. Let $U_{t}=\left(\begin{array}{ll}X_{t} & Y_{t} \\ Z_{t} & V_{t}\end{array}\right), t \geq 0$ be the solution of (2.2).

1. The process $\left(\begin{array}{l}X_{t} \\ Z_{t}\end{array}\right)_{t \geq 0}$ is a Brownian motion on $V_{n, k}$;

2. We have $\mathbb{P}\left(\inf \left\{t>0\right.\right.$, $\left.\left.\operatorname{det} Z_{t}=0\right\}<+\infty\right)=0$ and the process $\left(w_{t}\right)_{t \geq 0}:=\left(X_{t} Z_{t}^{-1}\right)_{t \geq 0}$ is a Brownian motion on $\widehat{G}_{n, k}$ with generator $\frac{1}{2} \Delta_{\widehat{G}_{n, k}}$, where

$$
\Delta_{\widehat{G}_{n, k}}=4 \sum_{1 \leq i, i^{\prime} \leq n-k, 1 \leq j, j^{\prime} \leq k}\left(I_{n-k}+w w^{*}\right)_{i i^{\prime}}\left(I_{k}+w^{*} w\right)_{j^{\prime} j} \frac{\partial^{2}}{\partial w_{i j} \partial \bar{w}_{i^{\prime} j^{\prime}}} .
$$

Proof. The first part of the theorem is straightforward to prove. Indeed, the map $\Pi: \mathbf{U}(n) \rightarrow V_{n, k},\left(\begin{array}{cc}X & Y \\ Z & V\end{array}\right) \rightarrow\left(\begin{array}{l}X \\ Z\end{array}\right)$ is a Riemannian submersion with totally geodesic fibers, therefore the process $\left(\Pi\left(U_{t}\right)\right)_{t \geq 0}$ is a Brownian motion on $V_{n, k}$. We now turn to the proof of the second part of the theorem. We first note that, as noticed above, $\left(Z_{t}^{*} Z_{t}\right)_{t \geq 0}$ is a matrix Jacobi process and therefore, from known properties of those processes, $\mathbb{P}\left(\inf \left\{t>0, \operatorname{det}\left(Z_{t}^{*} Z_{t}\right)=0\right\}<+\infty\right)=0$. We then turn to the study of $w=X Z^{-1}$ which is therefore well defined for all times. We need to introduce some notations. Let us consider the block decomposition

$$
A_{t}=\left(\begin{array}{cc}
\alpha_{t} & \beta_{t} \\
\gamma_{t} & \epsilon_{t}
\end{array}\right)
$$


Asymptotic windings of the block determinants of a unitary Brownian motion

with $\alpha_{t} \in \mathbb{C}^{k \times k}$. Note that $\alpha_{t}, \beta_{t}=-\gamma_{t}^{*}$ and $\epsilon_{t}$ are independent. From (2.2) we obtain the following system of stochastic differential equations:

$$
\begin{aligned}
& d X=X \circ d \alpha+Y \circ d \gamma=X d \alpha+Y d \gamma+\frac{1}{2}(d X d \alpha+d Y d \gamma) \\
& d Y=X \circ d \beta+Y \circ d \epsilon=X d \beta+Y d \epsilon+\frac{1}{2}(d X d \beta+d Y d \epsilon) \\
& d Z=Z \circ d \alpha+V \circ d \gamma=Z d \alpha+V d \gamma+\frac{1}{2}(d Z d \alpha+d V d \gamma) \\
& d V=Z \circ d \beta+V \circ d \epsilon=Z d \beta+V d \epsilon+\frac{1}{2}(d Z d \beta+d V d \epsilon) .
\end{aligned}
$$

Using then Itô's formula, long but routine computations yield

$$
d w=d\left(X Z^{-1}\right)=(Y d \gamma-w V d \gamma) Z^{-1} .
$$

From this expression, after further computations, we deduce

$$
\begin{aligned}
d w_{i j} d \bar{w}_{i^{\prime} j^{\prime}} & =\sum_{\ell, m=1}^{k}(Y-w V)_{i \ell}(\bar{Y}-\overline{w V})_{i^{\prime} m}\left(d \gamma Z^{-1}\right)_{\ell j}\left(d \bar{\gamma} \bar{Z}^{-1}\right)_{m j^{\prime}} \\
& =2\left((Y-w V)(Y-w V)^{*}\right)_{i i^{\prime}}\left(\left(Z Z^{*}\right)^{-1}\right)_{j^{\prime} j} d t \\
& =2\left(I_{n-k}+w w^{*}\right)_{i i^{\prime}}\left(\left(Z Z^{*}\right)^{-1}\right)_{j^{\prime} j} d t \\
& =2\left(I_{n-k}+w w^{*}\right)_{i i^{\prime}}\left(I_{k}+w^{*} w\right)_{j^{\prime} j} d t
\end{aligned}
$$

Therefore $\left(w_{t}\right)_{t \geq 0}$ is a diffusion with generator $\frac{1}{2} \Delta_{\widehat{G}_{n, k}}$. Then, to conclude, we note that it is indeed the Brownian motion on $\widehat{G}_{n, k}$ because $p$ is a Riemannian submersion with totally geodesic fibers.

Remark 2.2. If we consider on $\mathbb{C}^{(n-k) \times k}$ the probability measure

$$
d \mu:=c_{n, k} \operatorname{det}\left(I_{k}+w^{*} w\right)^{-n} d w
$$

with normalizing constant $c_{n, k}$, then a direct computation shows that $\mu$ is the symmetric and invariant measure for the diffusion $\left(w_{t}\right)_{t \geq 0}$, i.e. if $f, g$ are smooth and compactly supported functions on $\mathbb{C}^{(n-k) \times k}$, then

$$
\int_{\mathbb{C}^{(n-k) \times k}}\left(\Delta_{\widehat{G}_{n, k}} f\right) g d \mu=\int_{\mathbb{C}^{(n-k) \times k}} f\left(\Delta_{\widehat{G}_{n, k}} g\right) d \mu
$$

Remark 2.3. The complex Grassmannian $G_{n, k}$ is a compact irreducible symmetric space of rank $k$ and the complex Stiefel manifold is a Riemannian homogeneous space (but is not symmetric). As such, the Brownian motions on $G_{n, k}$ and $V_{n, k}$ and their distributions and pathwise properties can be studied using representation theory and stochastic differential geometry. The literature on those topics is nowadays quite substantial. We for instance refer to the early works by Eugene Dynkin [14, 15] and Paul \& Marie-Paule Malliavin [24] or more recent presentations like [25] and the book [16] (see in particular Chapter 8: Riemannian submersions and Symmetric spaces). In some sense, Theorem 2.1 provides a more pedestrian approach: We work in a specific choice of coordinates within the algebra of complex matrices and describe the $G_{n, k}$ and $V_{n, k}$ Brownian motions in those coordinates taking advantage of the additional structure given by the matrix multiplication. 
Asymptotic windings of the block determinants of a unitary Brownian motion

\subsection{Eigenvalue process}

In this section, for later use, we collect some properties of the eigenvalues of the process $\left(J_{t}\right)_{t \geq 0}:=\left(w_{t}^{*} w_{t}\right)_{t \geq 0}$ where $\left(w_{t}\right)_{t \geq 0}=\left(X_{t} Z_{t}^{-1}\right)_{t \geq 0}$ is a Brownian motion on $\widehat{G}_{n, k}$ as in Theorem 2.1. We note that

$$
J_{t}=w_{t}^{*} w_{t}=\left(Z_{t}^{-1}\right)^{*} X_{t}^{*} X_{t} Z_{t}^{-1}=\left(Z_{t} Z_{t}^{*}\right)^{-1}-I_{k}
$$

Therefore $\left(I_{k}+J\right)^{-1}=Z Z^{*}$, and the properties of $J$ and its eigenvalues can be deduced from the corresponding properties of the matrix Jacobi process $Z Z^{*}$ after basic algebraic manipulations. In particular, one immediately has the following result.

Lemma 2.4. Let $\left(J_{t}\right)_{t \geq 0}$ be given as above, then $\mathbb{P}\left(\inf \left\{t>0, \operatorname{det}\left(J_{t}\right)=0\right\}<+\infty\right)=0$ and there exists a Brownian motion $\left(\mathbf{B}_{t}\right)_{t \geq 0}$ in $\mathbb{C}^{k \times k}$ such that:

$$
d J=\sqrt{I_{k}+J} d \mathbf{B}^{*} \sqrt{I_{k}+J} \sqrt{J}+\sqrt{J} \sqrt{I_{k}+J} d \mathbf{B} \sqrt{I_{k}+J}+2(n-k+\operatorname{tr}(J))\left(I_{k}+J\right) d t
$$

We explicitly note that the proof of $\mathbb{P}\left(\inf \left\{t>0, \operatorname{det}\left(J_{t}\right)=0\right\}<+\infty\right)=0$ uses the fact that $k \leq n-k$.

Remark 2.5. The symmetric and invariant probability measure of the diffusion process $J$ can be easily obtained. Indeed, let $\mathbf{w}$ be a random variable on $\mathbb{C}^{(n-k) \times k}$ whose law is the probability measure with density $c_{n, k} \operatorname{det}\left(I_{k}+w^{*} w\right)^{-n} d w$. From Proposition 1 in [17] one has for every bounded Borel function $g$ and some normalization constant $c_{n, k}^{\prime}$ that

$$
\begin{aligned}
\mathbb{E}\left(g\left(\mathbf{w}^{*} \mathbf{w}\right)\right) & =c_{n, k} \int_{\mathbb{C}^{(n-k) \times k}} g\left(w^{*} w\right) \operatorname{det}\left(I_{k}+w^{*} w\right)^{-n} d w \\
& =c_{n, k}^{\prime} \int_{\hat{\mathcal{H}}_{k}} g(S) \operatorname{det}\left(I_{k}+S\right)^{-n} \operatorname{det}(S)^{n-2 k} d S .
\end{aligned}
$$

Thus, from Remark 2.2, the probability measure on the cone $\hat{\mathcal{H}}_{k}$ of positive definite Hermitian matrices with density $c_{n, k}^{\prime} \operatorname{det}\left(I_{k}+S\right)^{-n} \operatorname{det}(S)^{n-2 k} d S$ is the invariant and symmetric probability measure for the diffusion process $J$.

Concerning the eigenvalues of $J$ one obtains after applying techniques and results from $[11,13,18,19]$ the following result:

Lemma 2.6. Let $\lambda(t)=\left(\lambda_{1}(t), \ldots, \lambda_{k}(t)\right), t \geq 0$ be the eigenvalue process of the diffusion matrix $J$. Assume that $\lambda_{1}(0)>\cdots>\lambda_{k}(0)$, then the process $\lambda(t)$ is non colliding, i.e.

$$
\mathbb{P}\left(\forall t \geq 0, \lambda_{1}(t)>\cdots>\lambda_{k}(t)\right)=1 .
$$

Moreover, we have

$$
d \lambda_{i}=2\left(1+\lambda_{i}\right) \sqrt{\lambda_{i}} d B^{i}+2\left(1+\lambda_{i}\right)\left(n-2 k+1-(2 k-3) \lambda_{i}+2 \lambda_{i}\left(1+\lambda_{i}\right) \sum_{\ell \neq i} \frac{1}{\lambda_{i}-\lambda_{\ell}}\right) d t
$$

where $\left(B_{t}\right)_{t \geq 0}$ is a Brownian motion in $\mathbb{R}^{k}$.

Under the assumptions of the previous theorem, let us denote $\rho_{i}=\frac{1-\lambda_{i}}{1+\lambda_{i}}, i=1, \ldots, k$. Note that $\rho$ is the eigenvalue process of $2 Z Z^{*}-I_{k}$. Then, as an application of Itô's formula, we have

$$
d \rho_{i}=-2 \sqrt{1-\rho_{i}^{2}} d B^{i}-2\left(\left(n-2 k+(n-2 k+2) \rho_{i}\right)+2 \sum_{\ell \neq i} \frac{1-\rho_{i}^{2}}{\rho_{\ell}-\rho_{i}}\right) d t,
$$

where $\left(B_{t}\right)_{t \geq 0}$ is the same Brownian motion as in (2.6). Therefore, $\rho$ is a diffusion process with generator given by 
Asymptotic windings of the block determinants of a unitary Brownian motion

$$
\mathcal{L}_{n, k}=2 \sum_{i=1}^{k}\left(1-\rho_{i}^{2}\right) \partial_{i}^{2}-2 \sum_{i=1}^{k}\left(n-2 k+(n-2 k+2) \rho_{i}+2 \sum_{\ell \neq i} \frac{1-\rho_{i}^{2}}{\rho_{\ell}-\rho_{i}}\right) \partial_{i} .
$$

If we consider the Vandermonde function

$$
h(\rho)=\prod_{i>j}\left(\rho_{i}-\rho_{j}\right)
$$

then we have for every smooth function $f$ on $[-1,1]^{k}$ that

$$
\mathcal{L}_{n, k} f=2\left(\frac{1}{h} \mathcal{G}_{n-2 k, 0}(h f)+\frac{1}{6} k(k-1)(3 n-4 k+2) f\right) .
$$

where $\mathcal{G}_{a, b}:=\sum_{i=1}^{k}\left(1-\rho_{i}^{2}\right) \partial_{i}^{2}-\left(a-b+(a+b+2) \rho_{i}\right) \partial_{i}$ is a sum of Jacobi diffusion operators on $[-1,1]$. Therefore $(\rho(t))_{t \geq 0}$ is a Karlin-McGregor diffusion associated to a $k$-dimensional Jacobi process with independent components and conditioned by its ground state. Using then the well-known Karlin-McGregor formula, see [2], one deduces that for every $t>0, \rho(t)$ has a density with respect to the Lebesgue measure $d x$ given by

$$
e^{\frac{1}{3} k(k-1)(3 n-4 k+2) t} \frac{\prod_{i>j}\left(x_{i}-x_{j}\right)}{\prod_{i>j}\left(\rho_{i}(0)-\rho_{j}(0)\right)} \operatorname{det}\left(p_{t}^{n-2 k, 0}\left(\rho_{i}(0), x_{j}\right)\right)_{1 \leq i, j \leq k} \mathbf{1}_{\Delta_{k}}(x),
$$

where $p_{t}^{n-2 k, 0}$ is the transition density of a one-dimensional Jacobi diffusion (see (4.4) for a precise formula) and

$$
\Delta_{k}=\left\{x \in[-1,1]^{k},-1 \leq x_{1}<\cdots<x_{k} \leq 1\right\} .
$$

Moreover, when $t \rightarrow+\infty, \rho(t)$ converges in distribution to the invariant probability measure

$$
d \nu=c_{n, k} \prod_{1 \leq i<j \leq k}\left(x_{i}-x_{j}\right)^{2} \prod_{i=1}^{k}\left(1-x_{i}\right)^{n-2 k} \mathbf{1}_{\Delta_{k}}(x) d x,
$$

where $c_{n, k}$ is again a normalization constant and it might be explicitly computed using the well-known Selberg integral formula (see [1]).

\section{Skew-product decomposition of the Brownian motion of the Stiefel fibration}

\subsection{Connection form and horizontal Brownian motion on $V_{n, k}$}

Let us consider the Stiefel fibration

$$
\mathbf{U}(k) \rightarrow \widehat{V}_{n, k} \rightarrow \widehat{G}_{n, k}
$$

that was described in Sections 2.1 and 2.2. According to this fibration, one can see $\widehat{V}_{n, k}$ as a $\mathbf{U}(k)$-principal bundle over $\widehat{G}_{n, k}$. The next lemma gives a formula for the connection form of this bundle.

Lemma 3.1. Consider on $\widehat{V}_{n, k}$ the $\mathfrak{u}(k)$-valued one form

$$
\omega:=\frac{1}{2}\left(\left(X^{*} Z^{*}\right) d\left(\begin{array}{l}
X \\
Z
\end{array}\right)-d\left(X^{*} Z^{*}\right)\left(\begin{array}{l}
X \\
Z
\end{array}\right)\right)=\frac{1}{2}\left(X^{*} d X-d X^{*} X+Z^{*} d Z-d Z^{*} Z\right) .
$$

Then, $\omega$ is the connection form of the bundle $\mathbf{U}(k) \rightarrow \widehat{V}_{n, k} \rightarrow \widehat{G}_{n, k}$. 
Asymptotic windings of the block determinants of a unitary Brownian motion

Proof. We first observe that if $v=\left(\begin{array}{c}X \\ Z\end{array}\right) \in V_{n, k}$, then the tangent space to $V_{n, k}$ at $v$ is given by

$$
T_{v} V_{n, k}=\left\{\left(\begin{array}{l}
A \\
B
\end{array}\right) \in \mathbb{C}^{n \times k}, A^{*} X+X^{*} A+B^{*} Z+Z^{*} B=0\right\} .
$$

Then, if $\theta \in \mathfrak{u}_{k}$, one easily computes that the generator of the one-parameter group $\left\{q \rightarrow q e^{t \theta}\right\}_{t \in \mathbb{R}}$ is given by the vector field on $V_{n, k}$ whose value at $v$ is $\left(\begin{array}{l}X \theta \\ Z \theta\end{array}\right)$. Applying $\omega$ to this vector field yields $\theta$. To show that $\omega$ is the connection form it remains therefore to prove that the kernel of $\omega$ is the horizontal space of the Riemannian submersion $\left(\begin{array}{l}X \\ Z\end{array}\right) \rightarrow X Z^{-1}$. This horizontal space at $v$, say $\mathcal{H}_{v}$, is the orthogonal complement of the vertical space at $v$, which is the subspace $\mathcal{V}_{v}$ of $T_{v} V_{n, k}$ tangent to the fiber of the submersion. The previous argument shows that

$$
\mathcal{V}_{v}=\left\{\left(\begin{array}{c}
X \theta \\
Z \theta
\end{array}\right), \theta \in \mathfrak{u}(k)\right\}
$$

Therefore we have

$$
\mathcal{H}_{v}=\left\{\left(\begin{array}{l}
A \\
B
\end{array}\right) \in T_{v} V_{n, k}, \forall \theta \in \mathfrak{u}(k), \operatorname{tr}\left(A^{*} X \theta+B^{*} Z \theta\right)=0\right\} .
$$

We deduce from this that

$$
\mathcal{H}_{v}=\left\{\left(\begin{array}{l}
A \\
B
\end{array}\right) \in T_{v} V_{n, k}, A^{*} X+B^{*} Z=X^{*} A+Z^{*} B\right\},
$$

from which it is clear that $\omega_{\mid \mathcal{H}}=0$.

Our next goal is to describe the horizontal lift to $\widehat{V}_{n, k}$ of a Brownian motion on $\widehat{G}_{n, k}$. We still denote by $p: \widehat{V}_{n, k} \rightarrow \widehat{G}_{n, k}$ the Riemannian submersion. A continuous semimartingale $\left(M_{t}\right)_{t \geq 0}$ on $\widehat{V}_{n, k}$ is called horizontal if for every $t \geq 0, \int_{M[0, t]} \omega=0$, where $\int_{M[0, t]} \omega$ denotes the Stratonovich line integral of $\omega$ along the paths of $M$. If $\left(N_{t}\right)_{t \geq 0}$ is a continuous semimartingale on $\widehat{G}_{n, k}$ with $N_{0} \in \widehat{G}_{n, k}$, then if $\widetilde{N}_{0} \in \widehat{V}_{n, k}$ is such that $p\left(\widetilde{N}_{0}\right)=N_{0}$, there exists a unique horizontal continuous semimartingale $\left(\widetilde{N}_{t}\right)_{t \geq 0}$ on $\widehat{V}_{n, k}$ such that $p\left(\widetilde{N}_{t}\right)=N_{t}$ for every $t \geq 0$. The semimartingale $\left(\widetilde{N}_{t}\right)_{t \geq 0}$ is then called the horizontal lift at $\widetilde{N}_{0}$ of $\left(N_{t}\right)_{t \geq 0}$ to $\widehat{\widehat{V}}_{n, k}$. We refer to [5] or [16] for a more general description of the horizontal lift of a semimartingale in the context of foliations.

We then consider on $\widehat{G}_{n, k}$ the $\mathfrak{u}(k)$-valued one-form $\eta$ given by

$$
\begin{aligned}
\eta:= & \frac{1}{2}\left(\left(I_{k}+w^{*} w\right)^{-1 / 2}\left(d w^{*} w-w^{*} d w\right)\left(I_{k}+w^{*} w\right)^{-1 / 2}\right. \\
& \left.-\left(I_{k}+w^{*} w\right)^{-1 / 2} d\left(I_{k}+w^{*} w\right)^{1 / 2}+d\left(I_{k}+w^{*} w\right)^{1 / 2}\left(I_{k}+w^{*} w\right)^{-1 / 2}\right) .
\end{aligned}
$$

Theorem 3.2. Let $\left(w_{t}\right)_{t \geq 0}$ be a Brownian motion on $\widehat{G}_{n, k}$ started at $w_{0} \in \widehat{G}_{n, k}$ as in Theorem 2.1 and $\mathfrak{a}_{t}=\int_{w[0, t]} \eta$. Let $\left(\begin{array}{c}X_{0} \\ Z_{0}\end{array}\right) \in \widehat{V}_{n, k}$ be such that $X_{0} Z_{0}^{-1}=w_{0}$. The process

$$
\widetilde{w}_{t}:=\left(\begin{array}{c}
w_{t} \\
I_{k}
\end{array}\right)\left(I_{k}+w_{t}^{*} w_{t}\right)^{-1 / 2} \Theta_{t}
$$


Asymptotic windings of the block determinants of a unitary Brownian motion

is the horizontal lift at $\left(\begin{array}{c}X_{0} \\ Z_{0}\end{array}\right)$ of $\left(w_{t}\right)_{t \geq 0}$ to $\widehat{V}_{n, k}$, where $\left(\Theta_{t}\right)_{t \geq 0}$ is the $\mathbf{U}(k)$-valued solution of the Stratonovich stochastic differential equation

$$
\left\{\begin{array}{l}
d \Theta_{t}=\circ d \mathfrak{a}_{t} \Theta_{t} \\
\Theta_{0}=\left(Z_{0} Z_{0}^{*}\right)^{-1 / 2} Z_{0}
\end{array}\right.
$$

Proof. As before we denote by $p$ the submersion $\left(\begin{array}{l}X \\ Z\end{array}\right) \rightarrow X Z^{-1}$. It is easy to check that for every $t \geq 0, p\left(\widetilde{w}_{t}\right)=w_{t}$ and that $\widetilde{w}_{0}=\left(\begin{array}{c}X_{0} \\ Z_{0}\end{array}\right)$. It is therefore enough to prove that $\widetilde{w}$ is a horizontal semimartingale, i.e. that $\int_{\widetilde{w}[0, t]} \omega=0$. Denote

$$
X_{t}=w_{t}\left(I_{k}+w_{t}^{*} w_{t}\right)^{-1 / 2} \Theta_{t}, Z_{t}=\left(I_{k}+w_{t}^{*} w_{t}\right)^{-1 / 2} \Theta_{t}
$$

A long, but routine, computation shows that

$$
\begin{aligned}
& \frac{1}{2}\left(X^{*} \circ d X-\circ d X^{*} X+Z^{*} \circ d Z-\circ d Z^{*} Z\right) \\
= & -\frac{1}{2}\left(\circ d \Theta^{*} \Theta-\Theta^{*} \circ d \Theta+\Theta^{*}\left(\circ d\left(I_{k}+J\right)^{-1 / 2}\left(I_{k}+J\right)^{1 / 2}-\left(I_{k}+J\right)^{1 / 2} \circ d\left(I_{k}+J\right)^{-1 / 2}\right) \Theta\right. \\
& \left.+\Theta^{*}\left(I_{k}+J\right)^{-1 / 2}\left(\circ d w^{*} w-w^{*} \circ d w\right)\left(I_{k}+J\right)^{-1 / 2} \Theta\right) .
\end{aligned}
$$

where $J=w^{*} w$. Since $\circ d \Theta^{*}=\circ d \Theta^{-1}=-\Theta^{-1} \circ d \Theta \Theta^{-1}$ and $\circ d \Theta=\circ d \mathfrak{a} \Theta$ with

$$
\begin{aligned}
\circ d \mathfrak{a}= & \frac{1}{2}\left(I_{k}+J\right)^{-1 / 2}\left(\circ d w^{*} w-w^{*} \circ d w\right)\left(I_{k}+J\right)^{-1 / 2} \\
& -\frac{1}{2}\left(\left(I_{k}+J\right)^{-1 / 2} \circ d\left(I_{k}+J\right)^{1 / 2}-\circ d\left(I_{k}+J\right)^{1 / 2}\left(I_{k}+J\right)^{-1 / 2}\right)
\end{aligned}
$$

we conclude that

$$
\frac{1}{2}\left(X^{*} \circ d X-\circ d X^{*} X+Z^{*} \circ d Z-\circ d Z^{*} Z\right)=0
$$

and thus $\int_{\widetilde{w}[0, t]} \omega=0$.

\subsection{Skew-product decomposition of the Stiefel Brownian motion}

We now turn to the description of the Brownian motion on $\widehat{V}_{n, k}$ as a skew-product.

Theorem 3.3. Let $\left(w_{t}\right)_{t \geq 0}$ be a Brownian motion on $\widehat{G}_{n, k}$ started at $w_{0}=X_{0} Z_{0}^{-1} \in \widehat{G}_{n, k}$ as in Theorem 2.1 and let $\left(\Omega_{t}\right)_{t \geq 0}$ be a Brownian motion on the unitary group $\mathbf{U}(k)$ independent from $\left(w_{t}\right)_{t \geq 0}$. Let $\left(\Theta_{t}\right)_{t \geq 0}$ be the $\mathbf{U}(k)$-valued solution of the Stratonovich stochastic differential equation

$$
\left\{\begin{array}{l}
d \Theta_{t}=\circ d \mathfrak{a}_{t} \Theta_{t} \\
\Theta_{0}=\left(Z_{0} Z_{0}^{*}\right)^{-1 / 2} Z_{0}
\end{array}\right.
$$

where $\mathfrak{a}_{t}=\int_{w[0, t]} \eta$. The process

$$
\left(\begin{array}{c}
w_{t} \\
I_{k}
\end{array}\right)\left(I_{k}+w_{t}^{*} w_{t}\right)^{-1 / 2} \Theta_{t} \Omega_{t}
$$

is a Brownian motion on $\widehat{V}_{n, k}$ started at $\left(\begin{array}{c}X_{0} \\ Z_{0}\end{array}\right)$. 
Asymptotic windings of the block determinants of a unitary Brownian motion

Proof. We denote by $\Delta_{\mathcal{H}}$ the horizontal Laplacian and by $\Delta_{\mathcal{V}}$ the vertical Laplacian of the Stiefel fibration; see [4] for the definitions of horizontal and vertical Laplacians. Since the submersion $\widehat{V}_{n, k} \rightarrow \widehat{G}_{n, k}$ is totally geodesic, the operators $\Delta_{\mathcal{H}}$ and $\Delta_{\mathcal{V}}$ commute (see [4]). We note that the Laplace-Beltrami operator of $\widehat{V}_{n, k}$ is given by $\Delta_{\widehat{V}_{n, k}}=\Delta_{\mathcal{H}}+\Delta_{\mathcal{V}}$ and that the horizontal lift of the Brownian motion on $\widehat{G}_{n, k}$ is a diffusion with generator $\frac{1}{2} \Delta_{\mathcal{H}}$, see [5]. The fibers of the submersion $\widehat{V}_{n, k} \rightarrow \widehat{G}_{n, k}$ are isometric to $\mathbf{U}(k)$, thus if $f$ is a bounded Borel function on $\widehat{V}_{n, k}$, one has

$$
e^{\frac{1}{2} t \Delta \nu} f\left(\begin{array}{c}
X \\
Z
\end{array}\right)=\mathbb{E}\left(f\left(\begin{array}{c}
X \Omega_{t} \\
Z \Omega_{t}
\end{array}\right)\right) .
$$

Since $e^{\frac{1}{2} t \Delta_{\mathcal{V}}} e^{\frac{1}{2} t \Delta_{\mathcal{H}}}=e^{\frac{1}{2} t \Delta_{\widehat{V}_{n, k}}}$, we conclude from Theorem 3.2.

\section{Limit theorems}

Throughout the section, let $\left(w_{t}\right)_{t \geq 0}=\left(X_{t} Z_{t}^{-1}\right)_{t \geq 0}$ be a Brownian motion on $\widehat{G}_{n, k}$ where $\left(\begin{array}{l}X_{t} \\ Z_{t}\end{array}\right)_{t>0}$ is a Brownian motion on $\widehat{V}_{n, k}$. Our goal is to prove Theorem 1.1. Without loss of generality we will assume throughout the section that the eigenvalues of $Z_{0}^{*} Z_{0}$ are distinct; Even if the eigenvalues of the complex Jacobi process $Z_{t}^{*} Z_{t}$ are not distinct for $t=0$, they will be distinct for any $t>0$, see $[9,11]$, and thus from the Markov property, the limit Theorem 1.1 still holds.

\subsection{Main limit theorem}

We first give a limit theorem for the process $\left.\left(\int_{0}^{t} \operatorname{tr}\left(w_{s}^{*} w_{s}\right) d s\right)\right)_{t \geq 0}$ that shall be used in the next subsections. The method we use, a Girsanov transform, takes its root in the paper by M. Yor [30] and was further developed in the situation of matrix Wishart diffusions in [12] and in the situation of the real Jacobi matrix processes in Section 9.4.2 of the thesis [13]. Our result is the following:

Theorem 4.1. Let $\left(J_{t}\right)_{t \geq 0}=\left(w_{t}^{*} w_{t}\right)_{t \geq 0}$. The following convergence holds in distribution when $t \rightarrow+\infty$

$$
\frac{1}{t^{2}} \int_{0}^{t} \operatorname{tr}(J) d s \rightarrow X
$$

where $X$ is a random variable on $[0,+\infty)$ with density $\frac{k(n-k)}{\sqrt{2 \pi} x^{3 / 2}} e^{-\frac{k^{2}(n-k)^{2}}{2 x}}$ (therefore $X$ is the inverse of a gamma distributed random variable).

The proof is rather long and will be decomposed in several steps. We first recall that from Lemma 2.4, there exists a Brownian motion $\left(\mathbf{B}_{t}\right)_{t \geq 0}$ in $\mathbb{C}^{k \times k}$ such that:

$$
d J=\sqrt{I_{k}+J} d \mathbf{B}^{*} \sqrt{I_{k}+J} \sqrt{J}+\sqrt{J} \sqrt{I_{k}+J} d \mathbf{B} \sqrt{I_{k}+J}+2(n-k+\operatorname{tr}(J))\left(I_{k}+J\right) d t
$$

Lemma 4.2. We have

$$
d\left(\operatorname{det}\left(I_{k}+J\right)\right)=\operatorname{det}\left(I_{k}+J\right) \operatorname{tr}\left(\sqrt{J}\left(d \mathbf{B}+d \mathbf{B}^{*}\right)\right)+2 \operatorname{det}\left(I_{k}+J\right)(k(n-k)+\operatorname{tr}(J)) d t,
$$

and therefore

$$
d\left(\log \operatorname{det}\left(I_{k}+J\right)\right)=\operatorname{tr}\left(\sqrt{J}\left(d \mathbf{B}+d \mathbf{B}^{*}\right)\right)+2 k(n-k) d t .
$$

Proof. By Itô's formula we have

$$
d\left(\operatorname{det}\left(I_{k}+J\right)\right)=\sum_{i, j=1}^{k} \frac{\partial \operatorname{det}\left(I_{k}+J\right)}{\partial J_{i j}} d J_{i j}+\frac{1}{2} \sum_{i, j, i^{\prime}, j^{\prime}=1}^{k} \frac{\partial^{2} \operatorname{det}\left(I_{k}+J\right)}{\partial J_{i j} \partial J_{i^{\prime} j^{\prime}}} d J_{i j} d J_{i^{\prime} j^{\prime}} .
$$


Asymptotic windings of the block determinants of a unitary Brownian motion

First, we know that

$$
\frac{\partial \operatorname{det}(J)}{\partial J_{i j}}=\frac{\partial \sum_{\ell=1}^{k} J_{i \ell} \tilde{J}_{i \ell}}{\partial J_{i j}}=\tilde{J}_{i j}
$$

where $\tilde{J}=\operatorname{det}(J)\left(J^{T}\right)^{-1}$ is the cofactor of $J$. Hence the first order term writes $\operatorname{det}\left(I_{k}+\right.$ $J) \operatorname{tr}\left(\left(I_{k}+J\right)^{-1} d J\right)$. Next, we will use the following formula to compute the cross second order derivatives:

$\frac{\partial^{2} \operatorname{det}(J)}{\partial x \partial y}=(\operatorname{det}(J))\left(\operatorname{tr}\left(J^{-1} \frac{\partial^{2} J}{\partial x \partial y}\right)+\operatorname{tr}\left(J^{-1} \frac{\partial J}{\partial x}\right) \operatorname{tr}\left(J^{-1} \frac{\partial J}{\partial y}\right)-\operatorname{tr}\left(J^{-1} \frac{\partial J}{\partial x} J^{-1} \frac{\partial J}{\partial y}\right)\right)$.

Since $\frac{\partial J}{\partial J_{i j}}=E_{i j}$, clearly $\frac{\partial^{2} J}{\partial J_{i j} \partial J_{i^{\prime} j^{\prime}}}=0$. We also have $J^{-1} \frac{\partial J}{\partial J_{i j}}=\sum_{\ell}\left(J^{-1}\right)_{\ell i} E_{\ell j}$ and $\operatorname{tr}\left(J^{-1} \frac{\partial J}{\partial J_{i j}}\right)=\left(J^{-1}\right)_{j i}$. Hence

$$
\frac{\partial^{2} \operatorname{det}(J)}{\partial J_{i j} \partial J_{i^{\prime} j^{\prime}}}=(\operatorname{det}(J))\left(\left(J^{-1}\right)_{j i}\left(J^{-1}\right)_{j^{\prime} i^{\prime}}-\left(J^{-1}\right)_{j^{\prime} i}\left(J^{-1}\right)_{j i^{\prime}}\right)
$$

and

$$
\frac{\partial^{2} \operatorname{det}\left(I_{k}+J\right)}{\partial J_{i j} \partial J_{i^{\prime} j^{\prime}}}=\left(\operatorname{det}\left(I_{k}+J\right)\right)\left(\left(\left(I_{k}+J\right)^{-1}\right)_{j i}\left(\left(I_{k}+J\right)^{-1}\right)_{j^{\prime} i^{\prime}}-\left(\left(I_{k}+J\right)^{-1}\right)_{j^{\prime} i}\left(\left(I_{k}+J\right)^{-1}\right)_{j i^{\prime}}\right) .
$$

Moreover, from (4.1) we know that

$$
d J_{i j} d J_{i^{\prime} j^{\prime}}=2 d t\left(\left(J+J^{2}\right)_{i^{\prime} j}\left(I_{k}+J\right)_{i j^{\prime}}+\left(J+J^{2}\right)_{i j^{\prime}}\left(I_{k}+J\right)_{i^{\prime} j}\right)
$$

Hence we have

$$
\begin{aligned}
& d\left(\operatorname{det}\left(I_{k}+J\right)\right)=\operatorname{det}\left(I_{k}+J\right) \operatorname{tr}\left(\left(I_{k}+J\right)^{-1} d J\right) \\
& +\sum_{i, j, i^{\prime}, j^{\prime}=1}^{k} \operatorname{det}\left(I_{k}+J\right)\left(\left(\left(I_{k}+J\right)^{-1}\right)_{j i}\left(\left(I_{k}+J\right)^{-1}\right)_{j^{\prime} i^{\prime}}-\left(\left(I_{k}+J\right)^{-1}\right)_{j^{\prime} i}\left(\left(I_{k}+J\right)^{-1}\right)_{j i^{\prime}}\right) \\
& \quad \cdot\left(\left(J+J^{2}\right)_{i^{\prime} j}\left(I_{k}+J\right)_{i j^{\prime}}+\left(J+J^{2}\right)_{i j^{\prime}}\left(I_{k}+J\right)_{i^{\prime} j}\right) d t \\
& =\operatorname{det}\left(I_{k}+J\right) \operatorname{tr}\left(\left(I_{k}+J\right)^{-1} d J\right)-2(k-1) \operatorname{det}\left(I_{k}+J\right) \operatorname{tr}(J) d t .
\end{aligned}
$$

From (4.1) we know

$$
\operatorname{tr}\left(\left(I_{k}+J\right)^{-1} d J\right)=\operatorname{tr}\left(\sqrt{J}\left(d \mathbf{B}+d \mathbf{B}^{*}\right)\right)+2 k(n-k+\operatorname{tr}(J)) d t .
$$

Hence

$$
d\left(\operatorname{det}\left(I_{k}+J\right)\right)=\operatorname{det}\left(I_{k}+J\right) \operatorname{tr}\left(\sqrt{J}\left(d \mathbf{B}+d \mathbf{B}^{*}\right)\right)+2 \operatorname{det}\left(I_{k}+J\right)(k(n-k)+\operatorname{tr}(J)) d t
$$

As a direct consequence of $d\left\langle\operatorname{det}\left(I_{k}+J\right)\right.$, $\left.\operatorname{det}\left(I_{k}+J\right)\right\rangle=4 \operatorname{det}\left(I_{k}+J\right)^{2} \operatorname{tr}(J) d t$, we obtain (4.3) using Itô's formula.

Lemma 4.3. For every $\alpha \geq 0$ the process

$$
M_{t}^{\alpha}=e^{2 k \alpha(n-k) t}\left(\frac{\operatorname{det}\left(I_{k}+J_{0}\right)}{\operatorname{det}\left(I_{k}+J_{t}\right)}\right)^{\alpha} \exp \left(-2 \alpha^{2} \int_{0}^{t} \operatorname{tr}(J) d s\right)
$$

is a martingale. 
Asymptotic windings of the block determinants of a unitary Brownian motion

Proof. Consider the exponential local martingale

$$
M_{t}^{\alpha}:=\exp \left(-\alpha \int_{0}^{t} \operatorname{tr}\left(\sqrt{J}\left(d \mathbf{B}+d \mathbf{B}^{*}\right)\right)-2 \alpha^{2} \int_{0}^{t} \operatorname{tr}(J) d s\right),
$$

where $\mathbf{B}$ is the Brownian motion as given in Theorem 4.2. From Lemma 4.2, we have

$$
\left(\frac{\operatorname{det}\left(I_{k}+J_{t}\right)}{\operatorname{det}\left(I_{k}+J_{0}\right)}\right)^{\alpha}=\exp \left(\alpha\left(\int_{0}^{t} \operatorname{tr}\left(\sqrt{J}\left(d \mathbf{B}+d \mathbf{B}^{*}\right)\right)+2 k(n-k) d s\right)\right),
$$

and thus

$$
M_{t}^{\alpha}=e^{2 k \alpha(n-k) t}\left(\frac{\operatorname{det}\left(I_{k}+J_{0}\right)}{\operatorname{det}\left(I_{k}+J_{t}\right)}\right)^{\alpha} \exp \left(-2 \alpha^{2} \int_{0}^{t} \operatorname{tr}(J) d s\right) .
$$

From this expression, it is clear that there exists a constant $C>0$ such that we almost surely have $\left|M_{t}^{\alpha}\right| \leq C e^{2 k \alpha(n-k) t}$ and thus the process $\left(M_{t}^{\alpha}\right)_{t \geq 0}$ is a martingale.

In the next lemma, we provide a formula for the Laplace transform of the functional $\int_{0}^{t} \operatorname{tr}(J) d s$ using a Girsanov transform. In this computation, the transition kernel of one-dimensional Jacobi diffusions naturally appears. We recall the formula for this transition kernel. If we denote by $p_{t}^{a, b}(x, y)$ the transition density, with respect to the Lebesgue measure, of the diffusion with generator

$$
2\left(1-x^{2}\right) \frac{\partial^{2}}{\partial x^{2}}-2((a+b+2) x+a-b) \frac{\partial}{\partial x}
$$

and initiated from $x \in(-1,1)$, then we have

$$
p_{t}^{a, b}(x, y)=(1+y)^{b}(1-y)^{a} \sum_{m=0}^{+\infty} c_{m, a, b} e^{-2 m(m+a+b+1) t} P_{m}^{a, b}(x) P_{m}^{a, b}(y),
$$

where $c_{m, a, b}=\frac{2 m+a+b+1}{2^{a+b+1}} \frac{\Gamma(m+a+b+1) \Gamma(m+1)}{\Gamma(m+a+1) \Gamma(m+b+1)}$ and where $P_{m}^{a, b}(x), m \in \mathbb{Z}_{\geq 0}$ are the Jacobi polynomials given by

$$
P_{m}^{a, b}(x)=\frac{(-1)^{m}}{2^{m} m !(1-x)^{a}(1+x)^{b}} \frac{d^{m}}{d x^{m}}\left((1-x)^{a+m}(1+x)^{b+m}\right) .
$$

Lemma 4.4. For every $\alpha \geq 0$ and $t>0$

$$
\begin{aligned}
& \mathbb{E}\left(e^{-2 \alpha^{2} \int_{0}^{t} \operatorname{tr}(J) d s}\right) \\
& =C e^{\left(\frac{1}{3} k(k-1)(3 n-4 k+6 \alpha+2)-2 k(n-k) \alpha\right) t} \int_{\Delta_{k}} \operatorname{det}\left(\frac{p_{t}^{n-2 k, 2 \alpha}\left(\frac{1-\lambda_{i}(0)}{1+\lambda_{i}(0)}, x_{j}\right)}{\left(1+x_{j}\right)^{\alpha}}\right) \prod_{i, j}\left(x_{i}-x_{j}\right) d x .
\end{aligned}
$$

where

$$
C=\prod_{\ell} \frac{\left(1+\lambda_{\ell}(0)\right)^{\alpha}}{2^{\alpha}} \prod_{i>j} \frac{\left(1+\lambda_{i}(0)\right)\left(1+\lambda_{j}(0)\right)}{2\left(\lambda_{j}(0)-\lambda_{i}(0)\right)}
$$

is the normalization constant, $\lambda_{1}(0), \cdots, \lambda_{k}(0)$ are the ordered eigenvalues of $J_{0}, p_{t}^{n-2 k, 2 \alpha}$ is given by the formula (4.4) and

$$
\Delta_{k}=\left\{x \in[-1,1]^{k},-1 \leq x_{1}<\cdots<x_{k} \leq 1\right\} .
$$


Asymptotic windings of the block determinants of a unitary Brownian motion

Proof. Let $\alpha \geq 0$ and consider the probability measure $P^{\alpha}$ defined by

$$
\left.P^{\alpha}\right|_{\mathcal{F}_{t}}=\left.M_{t}^{\alpha} \cdot P\right|_{\mathcal{F}_{t}}
$$

We first note that

$$
\mathbb{E}\left(e^{-2 \alpha^{2} \int_{0}^{t} \operatorname{tr}(J) d s}\right)=e^{-2 k(n-k) \alpha t} \mathbb{E}^{\alpha}\left[\left(\frac{\operatorname{det}\left(I_{k}+J_{t}\right)}{\operatorname{det}\left(I_{k}+J_{0}\right)}\right)^{\alpha}\right]
$$

From Girsanov theorem, the process

$$
\beta_{t}=\mathbf{B}_{t}+2 \alpha \int_{0}^{t} \sqrt{J} d s
$$

is under $P^{\alpha}$ a $k \times k$-matrix-valued Brownian motion and we have

$d J=\sqrt{I_{k}+J} d \beta^{*} \sqrt{I_{k}+J} \sqrt{J}+\sqrt{J} \sqrt{I_{k}+J} d \beta \sqrt{I_{k}+J}+2(n-k-2 \alpha J+\operatorname{tr}(J))\left(I_{k}+J\right) d t$.

We now denote by $\lambda(t)=\left(\lambda_{i}(t)\right)_{1 \leq i \leq k}$ the eigenvalues of $J_{t}, t \geq 0$. From the previous equation satisfied by $J$ we deduce that there exists a Brownian motion $\left(B_{t}\right)_{t \geq 0}$ in $\mathbb{R}^{k}$ for the probability measure $P^{\alpha}$ such that

$d \lambda_{i}=2\left(1+\lambda_{i}\right) \sqrt{\lambda_{i}} d B^{i}+2\left(1+\lambda_{i}\right)\left(n-2 k+1-(2 k+2 \alpha-3) \lambda_{i}+2 \lambda_{i}\left(1+\lambda_{i}\right) \sum_{\ell \neq i} \frac{1}{\lambda_{i}-\lambda_{\ell}}\right) d t$.

Let us denote $\rho_{i}=\frac{1-\lambda_{i}}{1+\lambda_{i}}, i=1, \ldots, k$. Then, using Itô's formula and the previous equation, we have

$$
d \rho_{i}=-2 \sqrt{1-\rho_{i}^{2}} d B^{i}-2\left(\left(n-2 k-2 \alpha+(n-2 k+6 \alpha+2) \rho_{i}\right)+2 \sum_{\ell \neq i} \frac{1-\rho_{i}^{2}}{\rho_{\ell}-\rho_{i}}\right) d t .
$$

Using then the formula for the density of non-colliding Jacobi processes, see [2], we deduce that the process $(\rho(t))_{t \geq 0}$ has, under $P^{\alpha}$, a density with respect to the Lebesgue measure $d x$ given by

$$
e^{\frac{1}{3} k(k-1)(3 n-4 k+6 \alpha+2) t} \frac{\prod_{i>j}\left(x_{i}-x_{j}\right)}{\prod_{i>j}\left(\rho_{i}(0)-\rho_{j}(0)\right)} \operatorname{det}\left(p_{t}^{n-2 k, 2 \alpha}\left(\rho_{i}(0), x_{j}\right)\right)_{1 \leq i, j \leq k} \mathbf{1}_{\Delta_{k}}(x) .
$$

We conclude then with (4.5).

We are now ready for the proof of Theorem 4.1 .

Proof of Theorem 4.1. From Lemma 4.4, we have for every $\alpha \geq 0$ and $t>0$

$$
\begin{aligned}
& \mathbb{E}\left(e^{-2 \alpha^{2} \int_{0}^{t} \operatorname{tr}(J) d s}\right) \\
& =C e^{\left(\frac{1}{3} k(k-1)(3 n-4 k+6 \alpha+2)-2 k(n-k) \alpha\right) t} \int_{\Delta_{k}} \operatorname{det}\left(\frac{p_{t}^{n-2 k, 2 \alpha}\left(\frac{1-\lambda_{i}(0)}{1+\lambda_{i}(0)}, x_{j}\right)}{\left(1+x_{j}\right)^{\alpha}}\right) \prod_{i, j}\left(x_{i}-x_{j}\right) d x .
\end{aligned}
$$

In order to analyze the large time behavior of this Laplace transform we we will use the formula (4.4). We can write

$$
\begin{aligned}
& p_{t}^{n-2 k, 2 \alpha}(x, y) \\
= & (1+y)^{2 \alpha}(1-y)^{n-2 k} \sum_{m=0}^{+\infty} c_{m, n-2 k+2 \alpha} e^{-2 m(m+n-2 k+2 \alpha+1) t} P_{m}^{n-2 k, 2 \alpha}(x) P_{m}^{n-2 k, 2 \alpha}(y),
\end{aligned}
$$


Asymptotic windings of the block determinants of a unitary Brownian motion

Similarly to [11], or Section 3.9.1 in [2], denoting as before $\rho_{i}(0)=\frac{1-\lambda_{i}(0)}{1+\lambda_{i}(0)}$ we now compute

$$
\begin{aligned}
& \operatorname{det}\left(p_{t}^{n-2 k, 2 \alpha}\left(\rho_{i}(0), x_{j}\right)\right)_{1 \leq i, j \leq k} \\
= & \sum_{\sigma \in \mathfrak{S}_{k}} \operatorname{sgn}(\sigma) \prod_{i=1}^{k} p_{t}^{n-2 k, 2 \alpha}\left(\rho_{\sigma(i)}(0), x_{i}\right) \\
= & \sum_{\sigma \in \mathfrak{S}_{k}} \operatorname{sgn}(\sigma) \prod_{i=1}^{k}\left[\left(1-x_{i}\right)^{n-2 k}\left(1+x_{i}\right)^{2 \alpha}\right. \\
& \left.\times \sum_{m=0}^{+\infty} c_{m, n-2 k+2 \alpha} e^{-2 m(m+n-2 k+2 \alpha+1) t} P_{m}^{n-2 k, 2 \alpha}\left(\rho_{\sigma(i)}(0)\right) P_{m}^{n-2 k, 2 \alpha}\left(x_{i}\right)\right] \\
= & V_{\alpha}(x) \sum_{\sigma \in \mathfrak{S}_{k}} \operatorname{sgn}(\sigma) \\
& \times \sum_{m_{1}, \cdots, m_{k}=0}^{+\infty} \prod_{i=1}^{k} c_{m_{i}, n-2 k+2 \alpha} e^{-2 m_{i}\left(m_{i}+n-2 k+2 \alpha+1\right) t} P_{m_{i}}^{n-2 k, 2 \alpha}\left(\rho_{\sigma(i)}(0)\right) P_{m_{i}}^{n-2 k, 2 \alpha}\left(x_{i}\right)
\end{aligned}
$$

where $V_{\alpha}(x)=\prod_{i=1}^{k}\left(1-x_{i}\right)^{n-2 k}\left(1+x_{i}\right)^{2 \alpha}$. We can now write

$$
\begin{aligned}
& \sum_{\sigma \in \mathfrak{S}_{k}} \operatorname{sgn}(\sigma) \sum_{m_{1}, \cdots, m_{k}=0}^{+\infty} \prod_{i=1}^{k} c_{m_{i}, n-2 k+2 \alpha} e^{-2 m_{i}\left(m_{i}+n-2 k+2 \alpha+1\right) t} P_{m_{i}}^{n-2 k, 2 \alpha}\left(\rho_{\sigma(i)}(0)\right) P_{m_{i}}^{n-2 k, 2 \alpha}\left(x_{i}\right) \\
= & \sum_{m_{1}, \cdots, m_{k}=0}^{+\infty}\left(\prod_{i=1}^{k} c_{m_{i}, n-2 k+2 \alpha} e^{-2 m_{i}\left(m_{i}+n-2 k+2 \alpha+1\right) t} P_{m_{i}}^{n-2 k, 2 \alpha}\left(x_{i}\right)\right) \\
& \times \sum_{\sigma \in \mathfrak{S}_{k}} \operatorname{sgn}(\sigma) \prod_{i=1}^{k} P_{m_{i}}^{n-2 k, 2 \alpha}\left(\rho_{\sigma(i)}(0)\right) \\
= & \sum_{m_{1}, \cdots, m_{k}=0}^{+\infty}\left(\prod_{i=1}^{k} c_{m_{i}, n-2 k+2 \alpha} e^{-2 m_{i}\left(m_{i}+n-2 k+2 \alpha+1\right) t} P_{m_{i}}^{n-2 k, 2 \alpha}\left(x_{i}\right)\right) \\
& \times \operatorname{det}\left(P_{m_{i}}^{n-2 k, 2 \alpha}\left(\rho_{j}(0)\right)\right)_{1 \leq i, j \leq k} .
\end{aligned}
$$

By skew-symmetrization, we can rewrite the previous sum as

$$
\begin{aligned}
\sum_{m_{1}<\cdots<m_{k}} & \left(\prod_{i=1}^{k} c_{m_{i}, n-2 k+2 \alpha} e^{-2 m_{i}\left(m_{i}+n-2 k+2 \alpha+1\right) t}\right) \\
& \times \operatorname{det}\left(P_{m_{i}}^{n-2 k, 2 \alpha}\left(x_{j}\right)\right)_{1 \leq i, j \leq k} \operatorname{det}\left(P_{m_{i}}^{n-2 k, 2 \alpha}\left(\rho_{j}(0)\right)\right)_{1 \leq i, j \leq k} .
\end{aligned}
$$

Let us note that when $t \rightarrow+\infty$, the term of leading order in this sum corresponds to $\left(m_{1}, \cdots, m_{k}\right)=(0,1, \cdots, k-1)$ and is given by

$$
\begin{aligned}
\left(\prod_{i=1}^{k} c_{i-1, n-2 k+2 \alpha}\right) & e^{-\frac{1}{3} k(k-1)(3 n-4 k+6 \alpha+2) t} \\
& \times \operatorname{det}\left(P_{i-1}^{n-2 k, 2 \alpha}\left(x_{j}\right)\right)_{1 \leq i, j \leq k} \operatorname{det}\left(P_{i-1}^{n-2 k, 2 \alpha}\left(\rho_{j}(0)\right)\right)_{1 \leq i, j \leq k}
\end{aligned}
$$

On the other hand, from (4.7) one has for every $\lambda \geq 0$ and $t>0$ that

$$
\begin{aligned}
& \mathbb{E}\left(e^{-\frac{\lambda}{t^{2}} \int_{0}^{t} \operatorname{tr}(J) d s}\right) \\
= & C e^{\left(\frac{1}{3} k(k-1)\left(3 n-4 k+6 \frac{\sqrt{\lambda}}{\sqrt{2} t}+2\right)-2 k(n-k) \frac{\sqrt{\lambda}}{\sqrt{2} t}\right) t} \int_{\Delta_{k}} \operatorname{det}\left(\frac{p_{t}^{n-2 k, 2 \frac{\sqrt{\lambda}}{\sqrt{2} t}}\left(\rho_{i}(0), x_{j}\right)}{\left(1+x_{j}\right)^{\frac{\sqrt{\lambda}}{\sqrt{2} t}}}\right) \prod_{i, j}\left(x_{i}-x_{j}\right) d x .
\end{aligned}
$$


Asymptotic windings of the block determinants of a unitary Brownian motion

Using (4.8), one then deduces that for every $\lambda \geq 0$,

$$
\lim _{t \rightarrow+\infty} \mathbb{E}\left(e^{-\frac{\lambda}{t^{2}} \int_{0}^{t} \operatorname{tr}(J) d s}\right)=\tilde{C} e^{-k(n-k) \sqrt{2 \lambda}},
$$

where $\tilde{C}$ is a constant depending only on $n, k, \rho_{i}(0)$. For $\lambda=0, \mathbb{E}\left(e^{-\frac{\lambda}{t^{2}} \int_{0}^{t} \operatorname{tr}(J) d s}\right)=1$ and therefore $\tilde{C}=1$. One now concludes using an inverse Laplace transform that the following convergence takes place in distribution when $t \rightarrow+\infty$

$$
\frac{1}{t^{2}} \int_{0}^{t} \operatorname{tr}(J) d s \rightarrow X
$$

where $X$ is a random variable on $[0,+\infty)$ with density $\frac{k(n-k)}{\sqrt{2 \pi} x^{3 / 2}} e^{-\frac{k^{2}(n-k)^{2}}{2 x}}$. We incidentally note that $X$ is therefore distributed as the hitting time of $k(n-k)$ by a one-dimensional Brownian motion, even though this does not seem to be readily explainable.

\subsection{Asymptotics of a generalized stochastic area}

By the definition of the one-form $\eta$ in (3.3), we note that

$$
\begin{aligned}
\int_{w[0, t]} \operatorname{tr}(\eta) & =\frac{1}{2} \operatorname{tr}\left[\int_{0}^{t}\left(I_{k}+J\right)^{-1 / 2}\left(\circ d w^{*} w-w^{*} \circ d w\right)\left(I_{k}+J\right)^{-1 / 2}\right] \\
& =\frac{1}{2} \operatorname{tr}\left[\int_{0}^{t}\left(I_{k}+J\right)^{-1 / 2}\left(d w^{*} w-w^{*} d w\right)\left(I_{k}+J\right)^{-1 / 2}\right]
\end{aligned}
$$

where as before $J=w^{*} w$. From simple computations one can verify that

$$
\operatorname{tr}(d \eta)=\partial \bar{\partial} \log \operatorname{det}\left(I_{k}+w^{*} w\right),
$$

which implies that $i \operatorname{tr}(d \eta)$ is the Kähler form on $\widehat{G}_{n, k}$. Therefore $i \int_{w[0, t]} \operatorname{tr}(\eta)$ can be, in some sense, considered as a generalized stochastic area process on $\widehat{G}_{n, k}$; we refer to [6] for further explanation on the terminology of generalized stochastic area. In the proposition below we deduce large time limit distributions of such functionals.

Proposition 4.5. The following convergence holds in distribution when $t \rightarrow+\infty$

$$
\frac{1}{i t} \int_{w[0, t]} \operatorname{tr}(\eta) \rightarrow \mathcal{C}_{k(n-k)},
$$

where $\mathcal{C}_{k(n-k)}$ is a Cauchy distribution of parameter $k(n-k)$.

Proof. Using (2.4), similarly to the proof of (4.1) one can check that

$$
d w^{*} w-w^{*} d w=\sqrt{I_{k}+J} d \mathbf{B}^{*} \sqrt{I_{k}+J} \sqrt{J}-\sqrt{J} \sqrt{I_{k}+J} d \mathbf{B} \sqrt{I_{k}+J}
$$

where $\left(\mathbf{B}_{t}\right)_{t \geq 0}$ is a $k \times k$-matrix-valued Brownian motion. Therefore,

$$
\left(I_{k}+J\right)^{-1 / 2}\left(d w^{*} w-w^{*} d w\right)\left(I_{k}+J\right)^{-1 / 2}=d \mathbf{B}^{*} \sqrt{J}-\sqrt{J} d \mathbf{B}
$$

Consider then the diagonalization of $J=V \Lambda V^{*}$, where $V \in U(k)$ and $\Lambda=\operatorname{diag}\left\{\lambda_{1}, \ldots, \lambda_{k}\right\}$. We obtain

$$
d \mathbf{B}^{*} \sqrt{J}-\sqrt{J} d \mathbf{B}=V\left(V^{-1} d \mathbf{B}^{*} V \sqrt{\Lambda}-\sqrt{\Lambda} V^{-1} d \mathbf{B} V\right) V^{-1} .
$$

Therefore from (4.10), we have in distribution that

$$
\int_{w[0, t]} \operatorname{tr}(\eta)=i \mathcal{B}_{\int_{0}^{t} \operatorname{tr}(J) d s}
$$


Asymptotic windings of the block determinants of a unitary Brownian motion

where $\mathcal{B}$ is a one-dimensional Brownian motion independent from the process $\operatorname{tr}(J)$. Therefore, for every $\lambda>0$,

$$
\mathbb{E}\left(e^{-\lambda \frac{1}{i} \int_{w[0, t]} \operatorname{tr}(\eta)}\right)=\mathbb{E}\left(e^{-\lambda \mathcal{B} \int_{0}^{t} \operatorname{tr}(J) d s}\right)=\mathbb{E}\left(e^{-\frac{\lambda^{2}}{2} \int_{0}^{t} \operatorname{tr}(J) d s}\right) .
$$

We conclude then from (4.9) after straightforward computations

\subsection{Asymptotic windings}

We are now interested in the windings of the complex valued process $\operatorname{det}\left(Z_{t}\right)$. We first note that from Theorem 3.3, we have identity in law

$$
\operatorname{det}\left(Z_{t}\right)=\operatorname{det}\left(I_{k}+w_{t}^{*} w_{t}\right)^{-1 / 2} \operatorname{det} \Theta_{t} \operatorname{det} \Omega_{t} .
$$

We shall then use the following result.

Lemma 4.6. Let $G$ be a connected compact Lie group of $m \times m$ matrices with Lie algebra g. Let $\left(M_{t}\right)_{t \geq 0}$ be a $\mathfrak{g}$-valued continuous semimartingale such that $M_{0}=0$ and let $\left(C_{t}\right)_{t \geq 0}$ be the $G$-valued solution of the Stratonovich stochastic differential equation

$$
d C_{t}=\left(\circ d M_{t}\right) C_{t}
$$

Then, for $t \geq 0, \operatorname{det} C_{t}=\left(\operatorname{det} C_{0}\right) \exp \left(\operatorname{tr}\left(M_{t}\right)\right)$.

Proof. Let $T>0$. Consider on the time interval $[0, T]$ the sequence of $G$-valued semimartingales $\left(C_{t}^{n}\right)_{0 \leq t \leq T}$ inductively defined by

$$
C_{t}^{n}=C_{t_{k}}^{n} \exp \left(\frac{2^{n}}{T}\left(t-t_{k}\right)\left(M_{t_{k+1}}-M_{t_{k}}\right)\right), \quad t_{k} \leq t \leq t_{k+1},
$$

where $t_{k}=\frac{k T}{2^{n}}, k=0, \ldots, 2^{n}$. From Theorem 2 in [21], the sequence of semimartingales $\left(C_{t}^{n}\right)_{0 \leq t \leq T}$ converges in probability to $\left(C_{t}\right)_{0 \leq t \leq T}$ uniformly on $[0, T]$. However,

$$
\operatorname{det}\left(C_{t}^{n}\right)=\operatorname{det}\left(C_{t_{k}}^{n}\right) \exp \left(\frac{2^{n}}{T}\left(t-t_{k}\right) \operatorname{tr}\left(M_{t_{k+1}}-M_{t_{k}}\right)\right), \quad t_{k} \leq t \leq t_{k+1} .
$$

We deduce therefore by induction that

$$
\operatorname{det}\left(C_{T}^{n}\right)=\left(\operatorname{det} C_{0}\right) \exp \left(\operatorname{tr}\left(M_{T}\right)\right) .
$$

Letting then $n \rightarrow+\infty$ yields the conclusion.

Using the previous lemma, we deduce the following:

Lemma 4.7. For every $t \geq 0, \operatorname{det} \Theta_{t}=\frac{\operatorname{det} Z_{0}}{\left|\operatorname{det} Z_{0}\right|} \exp \left(\int_{w[0, t]} \operatorname{tr}(\eta)\right)$.

Proof. We have

$$
\left\{\begin{array}{l}
d \Theta_{t}=\circ d\left(\int_{w[0, t]} \eta\right) \Theta_{t} \\
\Theta_{0}=\left(Z_{0} Z_{0}^{*}\right)^{-1 / 2} Z_{0} .
\end{array}\right.
$$

Thus from Lemma 4.6 we have $\operatorname{det} \Theta_{t}=\frac{\operatorname{det} Z_{0}}{\left|\operatorname{det} Z_{0}\right|} \exp \left(\operatorname{tr}\left(\int_{w[0, t]} \eta\right)\right)$.

We are now finally in position to prove one of our main results. 
Asymptotic windings of the block determinants of a unitary Brownian motion

Theorem 4.8. One has the polar decomposition

$$
\operatorname{det}\left(Z_{t}\right)=\varrho_{t} e^{i \theta_{t}}
$$

where $0<\varrho_{t} \leq 1$ is a continuous semimartingale and $\theta_{t}$ is a continuous martingale such that the following convergence holds in distribution when $t \rightarrow+\infty$

$$
\frac{\theta_{t}}{t} \rightarrow \mathcal{C}_{k(n-k)}
$$

where $\mathcal{C}_{k(n-k)}$ is a Cauchy distribution of parameter $k(n-k)$.

Proof. From the decomposition $\operatorname{det}\left(Z_{t}\right)=\operatorname{det}\left(I_{k}+w_{t}^{*} w_{t}\right)^{-1 / 2} \operatorname{det} \Theta_{t} \operatorname{det} \Omega_{t}$ one $\operatorname{deduces}$ from Lemmas 4.6 and 4.7 that

$$
\varrho_{t}=\operatorname{det}\left(I_{k}+J_{t}\right)^{-1 / 2}, i \theta_{t}=i \theta_{0}+\operatorname{tr}\left(D_{t}\right)+\int_{w[0, t]} \operatorname{tr}(\eta)
$$

where $D_{t}$ is a Brownian motion on $\mathfrak{u}(k)$ independent from $w$ and $\theta_{0}$ is such that $e^{i \theta_{0}}=$ $\frac{\operatorname{det} Z_{0}}{\left|\operatorname{det} Z_{0}\right|}$. The conclusion follows then from Proposition 4.5.

Let us remark that it is also possible to compute the asymptotic law of the radial part $\varrho_{t}$. Indeed, from the previous proof, we know that $\varrho_{t}=\operatorname{det}\left(I_{k}+J_{t}\right)^{-1 / 2}$ and the limit distribution of the ordered eigenvalues of $\left(I_{k}-J\right)\left(I_{k}+J\right)^{-1}$ is computed explicitly in (2.7) to be a distribution with density

$$
c_{n, k} \prod_{1 \leq i<j \leq k}\left(x_{i}-x_{j}\right)^{2} \prod_{i=1}^{k}\left(1-x_{i}\right)^{n-2 k} \mathbf{1}_{\Delta_{k}}(x) d x .
$$

Using then the Selberg's integral formula, one obtains that in distribution one has

$$
\varrho_{t} \rightarrow \varrho_{\infty}
$$

where $\rho_{\infty}$ is a random variable such that for every $s \geq 0$

$$
\mathbb{E}\left(\varrho_{\infty}^{s}\right)=\tilde{c}_{n, k} \prod_{j=0}^{k-1} \frac{\Gamma\left(\frac{s}{2}+j+1\right)}{\Gamma\left(\frac{s}{2}+n-k+j+1\right)}
$$

where $\tilde{c}_{n, k}$ is a normalization constant. Thus, using uniqueness of the Mellin transform, one concludes that

$$
\varrho_{\infty}^{2}=\prod_{j=1}^{k} \mathfrak{B}_{j, n-k}
$$

where $\mathfrak{B}_{j, n-k}$ are independent beta random variables with parameters $(j, n-k)$. This recovers a result by A. Rouault (Proposition 2.4 in [26]).

\subsection{The case $k \geq n-k$}

In this section, we prove Theorem 1.1 in the case of $k \geq n-k$. Thus, unlike the rest of the paper, we assume in this section that $k \geq n-k$. This is essentially a duality argument equivalent to the isomorphism $G_{n, k} \simeq G_{n, n-k}$. Let

$$
U=\left(\begin{array}{ll}
X & Y \\
Z & V
\end{array}\right) \in \mathbf{U}(n)
$$


Asymptotic windings of the block determinants of a unitary Brownian motion

with $Z \in \mathbb{C}^{k \times k}, \operatorname{det}(Z) \neq 0$. Using $X^{*} X+Z^{*} Z=I_{k}$ and $X X^{*}+Y Y^{*}=I_{n-k}$ we deduce the following equality of spectrum

$$
\mathbf{s p}\left(Z^{*} Z\right)=\mathbf{s p}\left(Y Y^{*}\right) \cup\{1\}
$$

and that the eigenvalue 1 of $Z^{*} Z$ has multiplicity at least $2 k-n$. In particular, we have

$$
\operatorname{det}\left(Z^{*} Z\right)=\operatorname{det}\left(Y Y^{*}\right)
$$

and

$$
\operatorname{tr}\left[\left(Z Z^{*}\right)^{-1}-I_{k}\right]=\operatorname{tr}\left[\left(Y Y^{*}\right)^{-1}-I_{n-k}\right] .
$$

Consider now a Brownian motion

$$
U_{t}=\left(\begin{array}{cc}
X_{t} & Y_{t} \\
Z_{t} & V_{t}
\end{array}\right) \in \mathbf{U}(n)
$$

with $Z_{0} \in \mathbb{C}^{k \times k}, \operatorname{det}\left(Z_{0}\right) \neq 0$. The process $\left(U_{t}^{*}\right)_{t \geq 0}$ is also a Brownian motion on $\mathbf{U}(n)$. Therefore, when $t \rightarrow+\infty$,

$$
\left|\operatorname{det}\left(Z_{t}\right)\right|^{2}=\left|\operatorname{det}\left(Y_{t}^{*}\right)\right|^{2} \rightarrow \prod_{j=1}^{n-k} \mathfrak{B}_{j, k}
$$

For the study of the winding process of $\operatorname{det}\left(Z_{t}\right)$, we notice that in the case $k \leq n-k$ the only part of the proof of Theorem 4.8 that actually uses the fact that $k \leq n-k$ is the proof of Theorem 4.1 (in the case $k>n-k$ the stochastic differential equation for $J=w^{*} w$ is only defined up to the hitting time $\inf \left\{t \geq 0\right.$, det $\left.J_{t}=0\right\}<+\infty$ and the Girsanov transform method fails). To handle the case $k \geq n-k$, we note that $\operatorname{tr} J=\operatorname{tr}\left[\left(Z Z^{*}\right)^{-1}-I_{k}\right]=\operatorname{tr}\left[\left(Y Y^{*}\right)^{-1}-I_{n-k}\right]$. Since the process $\left(U_{t}^{*}\right)_{t \geq 0}$ is also a Brownian motion on $\mathbf{U}(n)$, one can use the argument of the proof of Theorem 4.1 to deduce that

$$
\frac{1}{t^{2}} \int_{0}^{t} \operatorname{tr}\left[\left(Y Y^{*}\right)^{-1}-I_{n-k}\right] d s \rightarrow X
$$

where $X$ is a random variable on $[0,+\infty)$ with density $\frac{k(n-k)}{\sqrt{2 \pi} x^{3 / 2}} e^{-\frac{k^{2}(n-k)^{2}}{2 x}}$. Therefore, the conclusion of Theorem 4.8 still holds in the case of $k \geq n-k$.

\subsection{On the moments of $\operatorname{det} Z_{t}$}

To conclude the paper with a possible view toward free probability that concerns the limit $n \rightarrow \infty$ with $k=\alpha n$ (see [10]) we show that it is possible to give a formula for the mixed moments of the process $\operatorname{det} Z_{t}$ that can be deduced from the previous computations.

Let

$$
U_{t}=\left(\begin{array}{cc}
X_{t} & Y_{t} \\
Z_{t} & W_{t}
\end{array}\right)
$$

be a Brownian motion on the unitary group $\mathbf{U}(n)$ with $Z_{t} \in \mathbb{C}^{k \times k}, 1 \leq k \leq n-1$. Assume that $\operatorname{det} Z_{0} \neq 0$. Let now $p, q \in \mathbb{N}$. As before, from the $\operatorname{decomposition} \operatorname{det}\left(Z_{t}\right)=$ $\operatorname{det}\left(I_{k}+w_{t}^{*} w_{t}\right)^{-1 / 2} \operatorname{det} \Theta_{t} \operatorname{det} \Omega_{t}$ one has from Lemmas 4.6 and 4.7 that

$$
\operatorname{det}\left(Z_{t}\right)=\varrho_{t} e^{i \theta_{t}}
$$

with

$$
\varrho_{t}=\operatorname{det}\left(I_{k}+J_{t}\right)^{-1 / 2}, i \theta_{t}=i \theta_{0}+\operatorname{tr}\left(D_{t}\right)+\int_{w[0, t]} \operatorname{tr}(\eta)
$$


Asymptotic windings of the block determinants of a unitary Brownian motion

where $D_{t}$ is a Brownian motion on $\mathfrak{u}(k)$ independent from $w$ and $\theta_{0}$ is such that $e^{i \theta_{0}}=$ $\frac{\operatorname{det} Z_{0}}{\left|\operatorname{det} Z_{0}\right|}$. Therefore, using the martingale of Lemma 4.3 and the Girsanov transform as in Lemma 4.4, we obtain:

$$
\begin{aligned}
\mathbb{E}\left(\left(\operatorname{det} Z_{t}\right)^{p}\left(\overline{\operatorname{det} Z_{t}}\right)^{q}\right) & =\mathbb{E}\left(\varrho_{t}^{p+q} e^{i(p-q) \theta_{t}}\right) \\
& =\mathbb{E}\left(\operatorname{det}\left(I_{k}+J_{t}\right)^{-(p+q) / 2} e^{i(p-q) \theta_{t}}\right) \\
& =e^{i(p-q) \theta_{0}-k(p-q)^{2} t} \mathbb{E}\left(\operatorname{det}\left(I_{k}+J_{t}\right)^{-(p+q) / 2} e^{(p-q) \int_{w[0, t]} \operatorname{tr}(\eta)}\right) \\
& =e^{i(p-q) \theta_{0}-k(p-q)^{2} t} \mathbb{E}\left(\operatorname{det}\left(I_{k}+J_{t}\right)^{-(p+q) / 2} e^{(p-q) i \mathcal{B}_{0}^{t} \operatorname{tr}(J) d s}\right) \\
& =e^{i(p-q) \theta_{0}-k(p-q)^{2} t} \mathbb{E}\left(\operatorname{det}\left(I_{k}+J_{t}\right)^{-(p+q) / 2} e^{-\frac{(p-q)^{2}}{2} \int_{0}^{t} \operatorname{tr}(J) d s}\right) \\
& =\frac{e^{i(p-q) \theta_{0}-k(p-q)^{2} t-k(n-k)|p-q| t}}{\operatorname{det}\left(I+J_{0}\right)^{\frac{1}{2}|p-q|}} \mathbb{E}^{\frac{1}{2}|p-q|}\left(\operatorname{det}\left(I_{k}+J_{t}\right)^{-\min (p, q)}\right)
\end{aligned}
$$

This last term can be computed because from the proof of Lemma 4.4, we have

$$
\begin{aligned}
& \mathbb{E}^{\frac{1}{2}|p-q|}\left(\operatorname{det}\left(I_{k}+J_{t}\right)^{-\min (p, q)}\right) \\
= & C e^{\left(\frac{1}{3} k(k-1)(3 n-4 k+3|p-q|+2)\right) t} \int_{\Delta_{k}} \operatorname{det}\left(\frac{p_{t}^{n-2 k,|p-q|}\left(\frac{1-\lambda_{i}(0)}{1+\lambda_{i}(0)}, x_{j}\right)}{\left(1+x_{j}\right)^{-\min (p, q)}}\right) \prod_{i, j}\left(x_{i}-x_{j}\right) d x .
\end{aligned}
$$

where $C$ is a normalization constant depending only on $p, q$ and the ordered eigenvalues of $J_{0}$, and $p_{t}^{n-2 k,|p-q|}$ is given by the formula (4.4). We note that if $q=0$ the formula simplifies considerably and yields

$$
\mathbb{E}\left(\left(\operatorname{det} Z_{t}\right)^{p}\right)=\left(\operatorname{det} Z_{0}\right)^{p} e^{-k p^{2} t-k(n-k) p t} .
$$

\section{References}

[1] Greg W. Anderson, Alice Guionnet, and Ofer Zeitouni, An introduction to random matrices, Cambridge Studies in Advanced Mathematics, vol. 118, Cambridge University Press, Cambridge, 2010. MR-2760897

[2] Theodoros Assiotis, Neil O'Connell, and Jon Warren, Interlacing Diffusions, arXiv e-prints (2016), arXiv:1607.07182.

[3] Christian Autenried and Irina Markina, Sub-riemannian geometry of stiefel manifolds, SIAM Journal on Control and Optimization 52 (2014), no. 2, 939-959. MR-3180839

[4] Fabrice Baudoin, Sub-Laplacians and hypoelliptic operators on totally geodesic Riemannian foliations, Geometry, analysis and dynamics on sub-Riemannian manifolds. Vol. 1, EMS Ser. Lect. Math., Eur. Math. Soc., Zürich, 2016, pp. 259-321. MR-3587668

[5] Fabrice Baudoin, Qi Feng, and Maria Gordina, Integration by parts and quasi-invariance for the horizontal Wiener measure on foliated compact manifolds, J. Funct. Anal. 277 (2019), no. 5, 1362-1422. MR-3969194

[6] Fabrice Baudoin and Jing Wang, Stochastic areas, winding numbers and hopf fibrations, Probability Theory and Related Fields 169 (2017), no. 3-4, 977-1005. MR-3719061

[7] Arthur L. Besse, Einstein manifolds, Ergebnisse der Mathematik und ihrer Grenzgebiete (3) [Results in Mathematics and Related Areas (3)], vol. 10, Springer-Verlag, Berlin, 1987. MR-0867684

[8] Benoît Collins, Antoine Dahlqvist, and Todd Kemp, The spectral edge of unitary Brownian motion, Probab. Theory Related Fields 170 (2018), no. 1-2, 49-93. MR-3748321

[9] Luc Deleaval and Nizar Demni, Moments of the Hermitian matrix Jacobi process, J. Theoret. Probab. 31 (2018), no. 3, 1759-1778. MR-3842169 
Asymptotic windings of the block determinants of a unitary Brownian motion

[10] N. Demni, Free Jacobi process, J. Theoret. Probab. 21 (2008), no. 1, 118-143. MR-2384475

[11] Nizar Demni, $\beta$-jacobi processes, Advances in Pure and Applied Mathematics 1 (2010), no. 3, 325-344. MR-2719370

[12] Catherine Donati-Martin, Yan Doumerc, Hiroyuki Matsumoto, and Marc Yor, Some properties of the Wishart processes and a matrix extension of the Hartman-Watson laws, Publ. Res. Inst. Math. Sci. 40 (2004), no. 4, 1385-1412. MR-2105711

[13] Yan Doumerc, Matrices aléatoires, processus stochastiques et groupes de réflexions, Ph.D. thesis, Toulouse 3, 2005.

[14] E. B. Dynkin, Non-negative eigenfunctions of the Laplace-Beltrami operator and Brownian motion in certain symmetric spaces, Dokl. Akad. Nauk SSSR 141 (1961), 288-291. MR0132607

[15] E. B. Dynkin, Brownian motion in certain symmetric spaces and the non-negative eigenfunctions of the Laplace-Beltrami operator, Izv. Akad. Nauk SSSR Ser. Mat. 30 (1966), 455-478. MR-0202206

[16] K. David Elworthy, Yves Le Jan, and Xue-Mei Li, The geometry of filtering, Frontiers in Mathematics, Birkhäuser Verlag, Basel, 2010. MR-2731662

[17] Peter J. Forrester, Matrix Polar Decomposition and Generalisations of the BlaschkePetkantschin Formula in Integral Geometry, arXiv e-prints (2017), arXiv:1701.04505. MR4060311

[18] David J. Grabiner, Brownian motion in a Weyl chamber, non-colliding particles, and random matrices, Ann. Inst. H. Poincaré Probab. Statist. 35 (1999), no. 2, 177-204. MR-1678525

[19] Piotr Graczyk and Jacek Małecki, Multidimensional yamada-watanabe theorem and its applications to particle systems, Journal of Mathematical Physics 54 (2013), no. 2, 021503. MR-3076363

[20] Piotr Graczyk and Jacek Małecki, Strong solutions of non-colliding particle systems, Electronic Journal of Probability 19 (2014). MR-3296535

[21] M. Hakim-Dowek and D. Lépingle, L'exponentielle stochastique des groupes de Lie, Séminaire de Probabilités, XX, 1984/85, Lecture Notes in Math., vol. 1204, Springer, Berlin, 1986, pp. 352-374. MR-942031

[22] Velimir Jurdjevic, Irina Markina, and F Silva Leite, Extremal curves on stiefel and grassmann manifolds, The Journal of Geometric Analysis (2019), 1-31. MR-4167270

[23] Thierry Lévy, Schur-Weyl duality and the heat kernel measure on the unitary group, Adv. Math. 218 (2008), no. 2, 537-575. MR-2407946

[24] Marie-Paule Malliavin and Paul Malliavin, Factorisations et lois limites de la diffusion horizontale au-dessus d'un espace riemannien symétrique, Théorie du potentiel et analyse harmonique (Exposés des Journées de la Soc. Math. France, Inst. Recherche Math. Avancée, Strasbourg, 1973), 1974, pp. 164-217. Lecture Notes in Math., Vol. 404. MR-0359023

[25] Pierre-Loïc Méliot, The cut-off phenomenon for Brownian motions on compact symmetric spaces, Potential Anal. 40 (2014), no. 4, 427-509. MR-3201989

[26] Alain Rouault, Asymptotic behavior of random determinants in the Laguerre, Gram and Jacobi ensembles, ALEA Lat. Am. J. Probab. Math. Stat. 3 (2007), 181-230. MR-2365642

[27] Frank Spitzer, Some theorems concerning 2-dimensional Brownian motion, Trans. Amer. Math. Soc. 87 (1958), 187-197. MR-0104296

[28] Yung-Chow Wong, Differential geometry of grassmann manifolds, Proceedings of the National Academy of Sciences of the United States of America 57 (1967), no. 3, 589. MR-0216433

[29] Yung-Chow Wong, Sectional curvatures of grassmann manifolds, Proceedings of the National Academy of Sciences of the United States of America 60 (1968), no. 1, 75. MR-0229173

[30] Marc Yor, Loi de l'indice du lacet brownien, et distribution de Hartman-Watson, Z. Wahrsch. Verw. Gebiete 53 (1980), no. 1, 71-95. MR-0576898 


\section{Electronic Journal of Probability Electronic Communications in Probability}

\section{Advantages of publishing in EJP-ECP}

- Very high standards

- Free for authors, free for readers

- Quick publication (no backlog)

- Secure publication $\left(\mathrm{LOCKSS}^{1}\right)$

- Easy interface (EJMS²)

\section{Economical model of EJP-ECP}

- Non profit, sponsored by $\mathrm{IMS}^{3}, \mathrm{BS}^{4}$, ProjectEuclid ${ }^{5}$

- Purely electronic

\section{Help keep the journal free and vigorous}

- Donate to the IMS open access fund ${ }^{6}$ (click here to donate!)

- Submit your best articles to EJP-ECP

- Choose EJP-ECP over for-profit journals

\footnotetext{
${ }^{1}$ LOCKSS: Lots of Copies Keep Stuff Safe http://www. lockss.org/

${ }^{2}$ EJMS: Electronic Journal Management System http://www.vtex.lt/en/ejms.html

${ }^{3}$ IMS: Institute of Mathematical Statistics http://www.imstat.org/

${ }^{4}$ BS: Bernoulli Society http://www. bernoulli-society.org/

${ }^{5}$ Project Euclid: https://projecteuclid.org/

${ }^{6}$ IMS Open Access Fund: http://www.imstat.org/publications/open.htm
} 